\title{
Performance Comparison of Different Dynamic Channel Allocation Techniques for Mobile Satellite Systems (1)
}

\author{
Enrico Del Re, Romano Fantacci, Giovanni Giambene \\ Dipartimento di Ingegneria Elettronica, Università degli Studi di Firenze \\ Via S. Marta 3, 50139 Firenze - Italy \\ Fantacci@cosimo.die.unifi.it
}

\begin{abstract}
Dynamic Channel Allocation (DCA) techniques permit a high resource utilization in cellular networks and are able to adapt themselves in the presence of rapid variations of traffic loads offered to the cells. Therefore, they are particularly suitable for Mobile Satellite Systems (MSSs). This paper compares the performance of several DCA solutions that are based on the evaluation of a cost function in terms of both quality of service parameters (i.e., blocking probabilities) and signaling load to be supported by the system. Both GEostationary Orbit (GEO) and Low Earth Orbit (LEO) MSSs have been considered. A particularly interesting DCA solution is proposed that tries to serve a new call attempt in a cell where no channel is available by means of a channel reconfiguration in an interfering cell. Handover requests that do not attain immediately service can be queued for a maximum time in order to enhance system performance.
\end{abstract}

\section{INTRODUCTION}

Future 3-rd generation mobile communication systems, denoted by the name "International Mobile Telecommunications after the year 2000" (IMT-2000), are expected to be addressed to a wide market spread everywhere in the world. In order to reach this expected diffusion, two important aspects are [1 - 5]:

- The realization of attracting services; this entails the implementation of multimedia and personalized services capable to meet the users' need; a subscriber will be identified by a universal personal number, regardless of both the terminal and the network he/she uses.

- The achievement of a global coverage so as to provide subscribers with mobile services anywhere and at anytime. The system globalization will be made possible through the interworking of several networks and, in particular through the integration of terrestrial cellular networks with Mobile Satellite Systems (MSSs), that are more suitable to cover scarcely populated regions.

In this paper, a future integrated scenario is considered, where terrestrial and satellite systems will harmonize in order to offer global high quality communication services. Various integrated solutions have been

(1) Work carried out under the financial support of MURST and ASI proposed in the literature [4]; here, we will refer to the ultimate and more complex level of integration for future IMT-2000, named "system integration":

- satellite and terrestrial cellular networks belong to a unique system,

- the same techniques as those of the terrestrial system (e.g., multi-access scheme, channel allocation protocols and mobility management, etc.) are adopted for the satellite system with a considerable technology reuse for the common parts (except RF equipment $\left({ }^{2}\right)$ ),

- the use of dual-mode mobile terminals allows rerouting procedures between terrestrial and satellite networks.

At present, R\&D efforts are addressed towards the definition of Mobile Satellite Systems (MSSs) that use a constellation of Non-GeoStationary Orbit (NGSO) satellites. A particularly attracting solution is represented by Low Earth Orbit satellites (LEO-MSSs), because they permit to relax the constraints on the link budget and allow the use of low-power hand-held mobile terminals [6 - 8]. Several LEO-MSSs have been proposed, e.g., IRIDIUM (by Motorola), GLOBALSTAR (by Loral Qualcomm Satellite Services Inc.), TELEDESIC (by

(2) The frequency bands allocuted to the terrestrial segment will be different from those assigned to the satellite segment of future IMT2000 system. 
Teledesic Corporation). As a reference scenario this paper considers the IRIDIUM system [6 - 8] which is formed by 66 satellites on 6 circular polar orbits at about $780 \mathrm{~km}$ of altitude. Each satellite covers a 48-cell-network with a multi-spot-beam antenna. This system is assumed as a working example, however the techniques here described can be applied to other MSSs as well.

Every time a mobile user with a call in progress leaves the coverage area of a spot-beam (= cell) and goes into an adjacent one, a procedure is started in order to provide the user with a new channel in the destination spot-beam (inter-beam handover). This procedure must be seamless, that is unnoticeable to the user.

With geostationary satellites we assume that cells are so wide on the earth that the probability of handover during call lifetime is negligible [9-11]. Whereas, if the satellite orbit is not geostationary (e.g., Low Earth Orbit, LEO, or Medium Earth Orbit, MEO) [10] cells are moving on the earth. The motion increases if the satellite altitude decreases [7]. So, if LEO satellites are considered, a cell rapidly crosses regions on the earth with different traffic load conditions and handover requests are highly frequent during call lifetime. These specific aspects require the investigation of suitable resource management techniques that are able to adapt themselves to rapid traffic load variations and to reduce the call dropping probability due to a handover failure. Moreover, a high number of handover requests means a heavy signaling load to be managed by the network entities.

We can note that future LEO-MSSs as well as terrestrial microcellular systems will be characterized by very high traffic variations both in time and in space and a very high number of handover requests during call lifetime; so, both systems should be based on similar resource management strategies in the light of the high level of integration expected for IMT-2000.

In this paper, a handover procedure is considered which is based on the signal quality received by the mobile user. According to a de-centralized implementation, the handover procedure is "mobile controlled" (3) [12]: when, on the basis of the perceived signal quality by the Mobile Station (MS), a suitable handover criterion is met (see below), the MS sends to the current satellite a handover request message. During this procedure all messages between the MS and the satellite system are exchanged via the old spot-beam; this is a backward handover procedure. The satellite must provide the MS with a new channel in the destination cell. Soon after the new path is activated, the old link is released, according to a make-before-break arrangement. Many handover criteria can be considered [13]; in case of MSSs and espe-

(3) In the LEO-MSS environment, the relative user-satellite motion is dominated by the satellite ground-track speed: the relative motion has "deterministic characteristics" that could be managed by the sarellite network in an automatic way: the sutellite could foresee when an active MS needs to be handed over a new cell. This entails that another possible implementation of the handover procedure could bo nerwork controlled [12]. cially for LEO-MSSs, the need for a handover must be quickly and timely revealed. Any delay in the handover decision may cause the call dropping due to a sudden reduction of the signal level. According to the classification made in [13], we consider that a suitable handover criterion should be based on a hysteresis margin: the MS continuously monitors the signal strength, $s$, received from the spot-beam currently managing it and compares this level with that relative to adjacent beams, $s_{i}$. The handover criterion is met when $s<s_{i}-h$ ( $h=$ hysteresis level). The hysteresis is used to avoid repeated handovers back and forth between the old spot and the new one, until this signal definitely overcomes the first one.

The channel allocation techniques compared in this paper are based on the resource reuse concept: two cells irradiated on the earth may simultaneously use the same channel provided that they are at a suitable distance $D$ that allows acceptable levels of co-channel interference; this distance is evaluated in the worst case.

Satellite systems are known to be power and/or bandwidth limited. Therefore, the satellite resource has to be carefully utilized. In order to cope with the need of a high performance resource management technique, we have considered Dynamic Channel Allocation (DCA) strategies [9 - 11, 14]: any channel can be assigned to any cell provided that the constraint on the reuse distance is fulfilled.

In this paper, a DCA solution is proposed which achieves improved performance by allowing a channel rearrangement at the call arrival instant. A channel rearrangement requires the switching of a call in progress in a cell from its current channel to another channel available in the same cell. This is an intra-beam handover procedure completely controlled by the network that is performed so as to pack the use of channels among the cells of the network.

Moreover, we refer to a distributed implementation for DCA techniques: each satellite with on board processing capabilities allocates channels without involving earth stations, on the basis of a table on the state of the resources; neighboring satellites communicate channel state information via Inter-Satellite-Links (ISLs) in order to globally coordinate the allocation.

Only voice services are considered in this paper. Then, calls that arrive at a cell where no channel is available are blocked and lost. The efficiency of channel allocation techniques is expressed in terms of quality of service parameters that are the following blocking probabilities:

- the blocking probability of new call attempts, $P_{b 1}$,

- the handover failure probability, $P_{b 2}$,

- the call dropping probability due to handover failure, $P_{\text {drop. }}$

- the probability of unsuccessful call due to either the blocking of the initial call attempt or the failure of a subsequent handover procedure, $P_{n s}$

It will be shown that, in terms of $P_{n s}$, the proposed DCA technique with channel rearrangements at the call 
arrival instants outperforms other resource management techniques found in the literature. Moreover, this technique will be also validated from the signaling load standpoint [15].

In order to highlight the impact of mobility on the performance of resource management techniques [16], two extreme mobility situations for MSSs have been envisaged: that is both GEO- and LEO- MSSs.

Finally, in LEO-MSSs, we have considered that, in addition to a channel allocation technique of the dynamic type, a suitable handover prioritization strategy will be essential in order to reduce the call dropping probability due to handover failure $[9-11,17-20]$.

This paper is organized as follows: section 2 describes the user mobility model assumed for LEO-MSSs and GEO-MSSs. In section 3, the strategies used to manage new call arrivals and handover requests have been outlined. Moreover, a particular technique to obtain that each cell of the simulated network has a complete belt of interfering cells has been described in section 4 . Then, section 5 presents the method used to evaluate the signaling load to be supported by the network for a resource management technique. Finally, section 6 deals with the performance comparison by simulations among the proposed dynamic channel allocation solutions both in GEO- and LEO- MSSs.

\section{THE MOBILITY MODEL}

The user mobility has a strong impact on the performance of the satellite communication networks under consideration. Hence, a suitable mobility model must be defined in order to compare different handover management strategies.

The impact of handovers on system performance depends on the orbit type chosen for the MSS (e.g., GEO, LEO).

In the GEO case, the cells illuminated by a satellite are fixed with respect to a point on the earth; moreover, the average call duration is negligible with respect to cell crossing times (according to MS speed). This means that the handover occurrence is very low and, therefore, it can be considered, with a good approximation, that a user does not change its cell during call lifetime (i.e., "fixed user") [10].

In LEO-MSSs, the satellite ground-track speed $V_{\text {trk }}$ is several orders of magnitude greater than the earth rotation speed and the user speed. Therefore, a convenient approximation is to represent the relative user-satellite motion only by the satellite ground-track speed [10]. Owing to the high value of $V_{\text {trk }}$ and the small cells, handover requests are highly frequent during call lifetime: the management of handover requests has a strong impact on the quality of service perceived by users (i.e., $P_{\text {drop }}$ ).

In defining the mobility model in the LEO-MSS case, we have called "source cell" the cell where the MS call begins and any cell reached by the related MS during the call lifetime has been named "transit cell". Let us refer to the following mobility model for LEO-MSSs (Figs. 1 and 2):

- MSs cross cells at a constant velocity (relative to the spot-beam footprint) equalling the satellite ground-track speed, i.e., vector $V_{\text {trk }}$.

- When a handover occurs, the destination cell is the neighboring cell in the direction of the satellite ground-track motion.

- From the call outset in a cell, an MS travels a distance in it that is obtained as follows (as if cells were square cells with side $2 R$, disposed according to a hexagonal layout; Fig. 2):

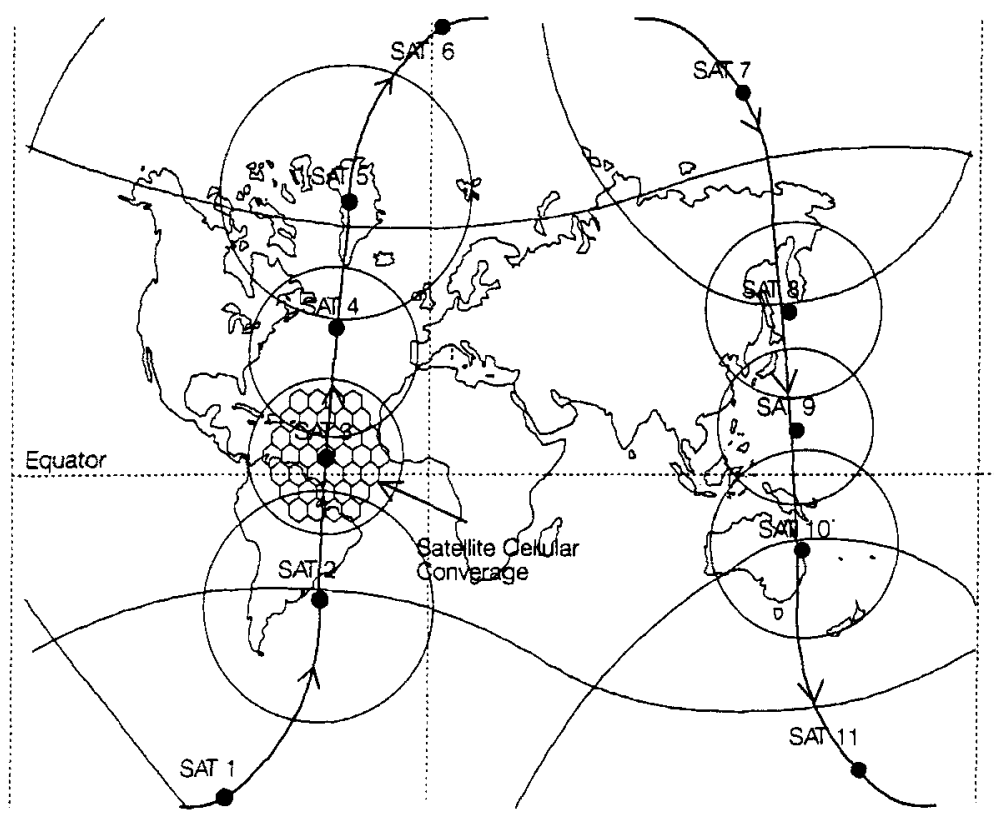

Fig. 1 - Satellites of a near-polar plane in an IRIDIUM-like LEO constellation. 


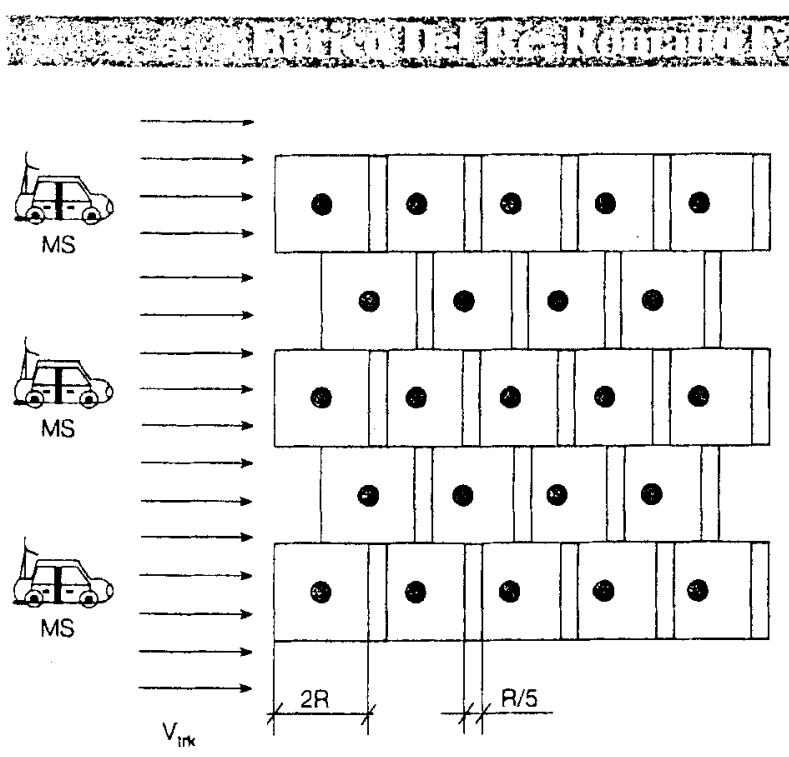

Fig. 2 - The hexagonal cellular layout with square cells and overlap areas used for the mobility model (LEO mobility case).

- uniformly distributed between 0 and $2 R$, if the cell is the "source cell" for the call;

- deterministically equal to $2 R$, if the call is in a "transit cell".

If the MS reaches the border with an adjacent cell with a call in progress, a handover procedure is immediately started (i.e., we assume that the handover criterion is met).

Further assumptions that complete this model will be given in section 3 - part 3.2. about the extension of the overlap areas between adjacent cells.

The user mobility will be characterized by the dimensionless parameter $\alpha$, defined as:

$\alpha \triangleq \frac{2 R}{V_{\mathrm{trk}} T_{m}}$

where $T_{m}$ represents the average call duration.

Further details about the mobility model outlined above are given in [10]. In particular, from [10] we obtain that the average number of handover requests per call is equal to $1 / \alpha$ for $P_{b 1}=P_{b 2}=0$; this is a geometrical parameter that gives an upper bound to the frequency of handover requests during call lifetime.

\section{A DYNAMIC ChANNEL Allocation TECHNIQUE FOR LEO-MSSS}

In this section, a DCA technique is proposed which efficiently manages both new call attempts and handover requests. Let us assume that, if a channel is used in a given cell $x$, it cannot be re-used in some tiers of cells (typically, one or two tiers) around that cell: this is the reuse distance constraint. These cells form the belt of interfering cells of $x, I(x)$. Let us denote by $\Lambda(x)$, the set of available channels for cell $x$ at the call arrival instant in $x$, i.e., those channels not used both in $x$ and in $I(x)$ at that time.

According to a distributed DCA implementation, each satellite manages a table on the state of the channels both for the cells it irradiates and for the interfering cells which belong to adjacent satellites [21]. The table has to be updated every time a channel state change occurs in that cells. A satellite is assumed to communicate channel state changes in the cells it generates to adjacent interfering satellites through ISLs.

\subsection{Management of new call attempts}

Let us assume that a new call arrival must be served in cell $x$ :

$\square$ if it results $\Lambda(x) \neq \emptyset$, the channel to be allocated in $x$, $i^{*}$, is selected within $\Lambda(x)$ according to the following minimum cost criterion:

$C_{x}\left(i^{*}\right)=\min _{i \in \Lambda(x)}\left[C_{x}(i)\right]$

where the cost function $C_{x}(i)$ will be defined at the end of point 3.1. in this section.

If more channels verify (2), a random choice is performed to obtain channel $i^{*}$.

The DCA technique previously proposed in $[9-10]$ is only composed by this first $\square$ step, because it blocks new call arrivals if $\Lambda(x)=\emptyset$. The new algorithm under consideration permits to serve new call arrivals, even if $\Lambda(x)=$ $\varnothing$, by means of the "Persistent Polite Aggressive" (PPA) approach outlined below [14]. Therefore, the allocation strategy composed by the previous and the following steps is denoted by PPA-DCA.

$\square$ If it results $\Lambda(x)=\varnothing$, the PPA mechanism starts its search to define the elements (if any) of the set of couples $\Omega(x)=[(\beta, \delta)]$, where the generic couple $(\beta$, $\delta$ ) represents a channel $\beta$ that results locked in cell $x$ only by its use in a cell $\delta \in I(x)$. Within $\Omega(x)$, we exclude the couples $(\beta, \delta)$, where cell $\delta$ has no available channel (i.e., $\Lambda(\delta)=\varnothing$ ):

$\Gamma(x)=[(\beta, \delta) \in \Omega(x): \Lambda(\delta) \neq \varnothing]$

- If $\Gamma(x) \neq \varnothing$, each couple $(\beta, \delta) \in \Gamma(x)$ represents a possible channel rearrangement (i.e., an intrabeam handover) in an interfering cell of $x$ in order to unlock a channel in $x$. The most convenient channel rearrangement, characterized by the couple $\left(\beta^{*}, \delta^{*}\right) \in \Gamma(x)$, is obtained according to the two following steps:

- selection of the cell $\delta^{*}$, among the cells $\mathrm{d}$ in the couples $\left({ }^{4}\right)(\beta, \delta) \in \Gamma(x)$ :

$\delta^{*}:|| \Lambda\left(\delta^{*}\right)||=\max _{\Gamma(x)}|| \Lambda(\delta)||$

$\left.{ }^{(}\right)$Note that many channels $\beta_{i}$ may be associated to the same cell $d$ so that couples $\left(\beta_{i}, \delta\right)$ belong to $\Gamma(x)$. 
where the symbol $\|$. II denotes the cardinality of a set.

If more couples in $(\beta, \delta) \in \Gamma(x)$ fulfil the condition (4), a random choice is performed to select the cell $\delta^{*}$.

Note that the criterion expressed by (4) selects $\delta^{*}$ as the cell with the greatest number of available channels: in this cell we have the greatest number of possible channel rearrangements from an allocated channel to another available channel.

- selection of channel $\beta^{*}$ to be released in cell $\delta^{*}$ among those channels $\beta$ which fulfil $\left(\beta, \delta^{*}\right) \in$ $\Gamma(x)$, on the basis of the channel de-allocation minimum cost criterion described at the point 3.3. of this section [10].

Then, channel $\beta^{*}$ is de-allocated in cell $\delta^{*}$ and the call in progress on $\beta^{*}$ is rearranged on another channel chosen in the set $\Lambda\left(\delta^{*}\right)-\left\{\beta^{*}\right\}$, according to the minimum cost criterion outlined at the first $\square$ step of this section. Then, channel $\beta^{*}$ becomes available in cell $x$ and it is assigned to the new call arrival.

- If $\Gamma(x)=\varnothing$, the new call arrival in cell $x$ is blocked and lost.

This algorithm is "polite-aggressive", since it tries to serve a new call arrival (otherwise blocked) by allowing a channel rearrangement in an interfering cell. Moreover, this technique is also "persistent", because it searches a channel reconfiguration by considering each possibility given by the set $\Omega(x)$, without limiting itself to only one or two attempts.

Each satellite with on board processing capabilities can easily manage the tasks required by the PPA-DCA algorithm on the basis of the channel state table. In the following, it will be proved that the increase in the sigraling load due to the use of the PPA policy in the allocation algorithm (i.e., the second $\square$ step) is negligible and can be supported by the system.

The cost function $C_{x}(i)$ used in (2) for the channel selection is described below [10]. We start by considering the channels allocated according to a Fixed Channel Allocation (FCA); let $F_{D}(x)$ be the set of channels allocated to cell $x$ according to FCA. A fixed allocation assures a distribution of channels among the cells of the network, with the minimum possible reuse distance, $D$. In the following, we define the cost function so as to allocate channels according to the regular FCA pattern, whenever possible.

The allocation cost contribution for channel $i \in \Lambda(x)$, due to the interfering cell $k \in I(x), C_{x}(k, i)$, can be expressed as:

$C_{r}(k, i) \wedge u_{k}(i)+2\left[1-q_{k}(i)\right], \forall k \in I(x)$

Where $u_{k}(i)$ and $q_{k}(i)$ are given by:

$$
\begin{aligned}
& u_{k}(i)= \begin{cases}1, & \text { if } i \in \Lambda(k) \\
0, & \text { otherwise }\end{cases} \\
& q_{k}(i)= \begin{cases}0, & \text { if } i \in F_{D}(k) \\
1, & \text { otherwise }\end{cases}
\end{aligned}
$$

In defining the cost contribution $C_{x}(k, i)$, relative to the status of channel $i$ in cell $k \in I(x)$, the first term takes into account the availability of channel $\mathrm{i}$ in cell $k$, while the second term takes into account if channel $i$ belongs to the optimal set for cell $k$ (i.e., $F_{D}(k)$ ). In addition to this, we have chosen to weight this second term by a factor 2 , in order to follow as long as possible the FCA channel distribution.

Therefore, the overall cost function can be obtained as:

$$
C_{x}(i) \triangleq q_{x}(i)+\sum_{k \in I(x)}\left[C_{x}(k, i)\right], \quad \forall i \in \Lambda(x)
$$

where the term $q_{x}(i)$ is introduced in (7) to discriminate among different situations with equal cost contributions for the interfering cells, because it is preferable to allocate in $x$ a channel $i$ belonging to the nominal (FCA) channel set of $x$ (i.e., $i \in F_{D}(x)$ ).

The PPA-DCA algorithm used to manage new call attempts has been summarized in Fig. 3. In order to clarify how the PPA-DCA technique operates, we refer to the example shown in Fig. 4, where we have assumed that the reuse distance $D$ is equal to $3 R$ and that the system has 6 channels to serve call demands in the cells; so, according to the FCA pattern, each cell has two nominal channels as shown in Fig. 4, where the symbols $\diamond 1, \diamond 2, \diamond 3, \ldots$ denote channels $\# 1, \# 2, \# 3, \ldots$. in use in the associated cells.

Let us assume that a new call attempt arrives at cell $x$ : in this cell we have $\Lambda(x)=\{\varnothing\}$, that is, no channel is available. Therefore, if we use the DCA algorithm the channel demand in cell $x$ is blocked and lost. Then, we apply the PPA-DCA technique in order to serve (if possible) the call in cell $x$ by means of a channel rearrangement in an interfering cell.

The set $\Omega(x)=[(\# 3, f) ;(\# 4, f) ;(\# 5, c) ;(\# 6, c)]$ contains the couples $(\beta, \delta)$, where, if channel $\beta$ is released in cell $\delta$, it becomes available in cell $x$. Channel rearrangements in the cells belonging to $\Omega(x)$ can be performed only in those cells with available channels; in this case, both cell $c$ and cell $f$ have available channels: $\Lambda(c)=\{\# 3, \# 4\}, \Lambda(f)=\{\# 6\}$. Then, $\Gamma(x) \equiv \Omega(x)$. Since the set $\Gamma(x) \neq \varnothing$, it is possible to find a channel rearrangement in an interfering cell of $x$ that is able to unlock a channel in cell $x$.

Within all possibilities represented by the elements of $\Gamma(x)$, we must choose the most convenient cell, $\delta^{*}$, between cell $c$ and cell $f$ and, within this cell $\delta^{*}$, the most convenient channel, $\beta^{*}$. In this case, the selected cell $\delta^{*} \equiv c$, because cell $c$ has more channels available than cell $f$ and may guarantee a greater number of pos- 


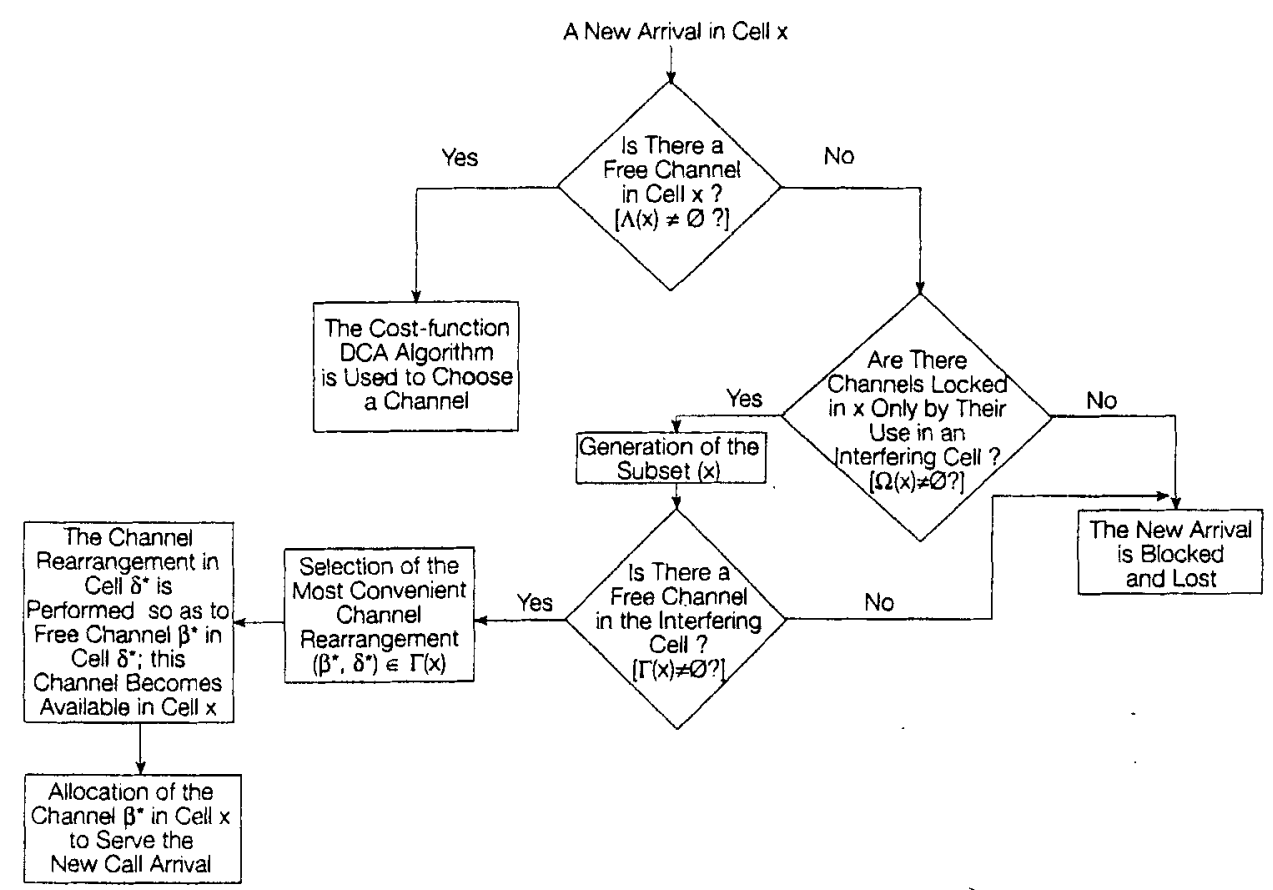

Fig. 3 - The policy used to manage the new arrival attempts by PPA-DCA.

sibilities for channel rearrangements. Then, in cell $c$ either channel \#5 or channel \#6 can be released; we select channel \#6 according to the de-allocation cost function criterion outlined at the point $C$ (i.e., selection of the most convenient channel to be released in cell $c$ [10]). Moreover, in cell $c$ the call in progress on channel \#6 must be rearranged on another channel available in cell $c$, that is either channel \#3 or channel \#4. We select channel \#3 to be assigned to cell $c$ according to the allocation cost function criterion explained in this point 3.1. (i.e., selection of the most convenient channel to be used in cell $c$ among those available, except channel \#6 [10]).

In conclusion, the call in progress in cell $c$ on channel \#6 is rearranged on channel \#3; therefore, channel \#6 becomes available to serve the new call arrival in cell $x$.

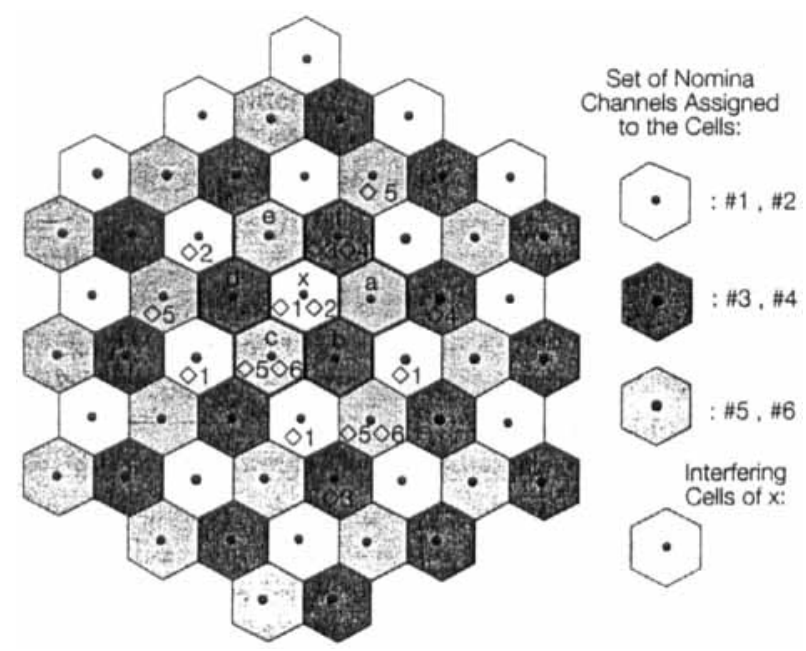

Fig. 4 - An illustrating example for the PPA-DCA algorithm.
The PPA-DCA algorithm operates a better channel packing than the simple DCA algorithm, by means of an improved channel distribution among the cells of the network; the PPA-DCA algorithm permits to serve also calls that are blocked by the DCA technique.

\subsection{MANAGEMENT OF HANDOVER REQUESTS}

Let us assume that an active MS is leaving cell $x$ and it is going into an adjacent cell $y$; in this new cell, the MS must be provided with a new channel to carry on the communication. A free channel is selected in cell $y$ according to the basic DCA algorithm (i.e., only the first $\square$ step of the previous point 3.1.); then, a channel must be released in cell $x$ (see the next point 3.3.). If no channel is immediately available in cell $y$, this procedure is delayed. We assume that there is a certain overlap among adjacent beam footprints on the earth; an MS can receive the signal from adjacent beams in an overlap area. Therefore, if all channels are busy in the destination cell of MS, the handover is queued, waiting for an available channel for all the time $t_{\text {wmax }}$ (the maximum queueing time) the MS spends to cross the overlap area. In the meantime, the MS call is still served by the originating cell, according to a make-before-break handover procedure. Elapsed $t_{w \max }$, if the handover has not been performed and the call is still in progress, the handover procedure fails and the related call is dropped.

Let us assume that an MS sends a handover request to the satellite as soon as it enters the overlap area between cell $x$ and cell $y$. Moreover, let us consider that a new call attempt that originates in the overlap area is immediately addressed towards the destination cell in order to avoid that it needs a handover soon after being served. 
In a real situation, the overlap area extension $\left(^{5}\right)$ and then $t_{\text {wmax }}$ are random variables that depend on several parameters, such as the MS (relative to the spot-beam footprint) motion direction, the satellite antenna characteristics and the propagation conditions; however, for the sake of simplicity, we will consider a deterministic extension of the overlap area, equal to $R / 5$ (conservative choice [10]). Then, according to the mobility assumptions made in section 2 , the time $t_{\text {wmax }}$ spent by an MS to cross this area with speed $V_{\text {trk }}$ results in:

$t_{w \max }=\frac{\alpha T_{m}}{10}$

A handover request sent by an MS is served according to the following steps:

$\square$ if it results $\Lambda(y) \neq \varnothing$, the handover procedure is immediately performed: a new channel is assigned to the call in cell $y$, according to the basic DCA strategy outlined at the first $\square$ step of the previous point 3.1. on the channel allocation strategy; then, the old channel is released in cell $x$ (see the point 3.3. of this section);

$\square$ if it results $\Lambda(y)=\emptyset$, the handover request is queued waiting for an available channel in cell $y$ (according to the basic DCA technique). In the meantime, the communication is served by the originating cell. We have assumed that the queueing discipline is of the
FIFO type (6). A handover request leaves the queue owing to one of the three following reasons:

- The handover procedure is successful: the handover request is performed (i.e., a channel becomes free in cell $y$ ), before the call is ended and its maximum queueing time has expired.

- The handover request declines: the associated call ends before the corresponding handover request is served and its maximum queueing time has expired.

- The handover procedure fails and the associated call is dropped: the handover has not been performed within $t_{w \max }$ and the call is not ended before its maximum queueing time has expired.

The handover queueing strategy has been summarized in Fig. 5.

\subsection{Management of call terminations}

In order to improve the performance of the DCA technique, we propose that, whenever a call termination occurs in a cell $x$ (due to either the end of the call or an inter-beam handover), the most convenient channel is released in cell $x$; the channel to be released is chosen on the basis of a de-allocation cost function complementary to that defined at point 3.1. to allocate new call arrivals. If the most convenient channel to be released is different

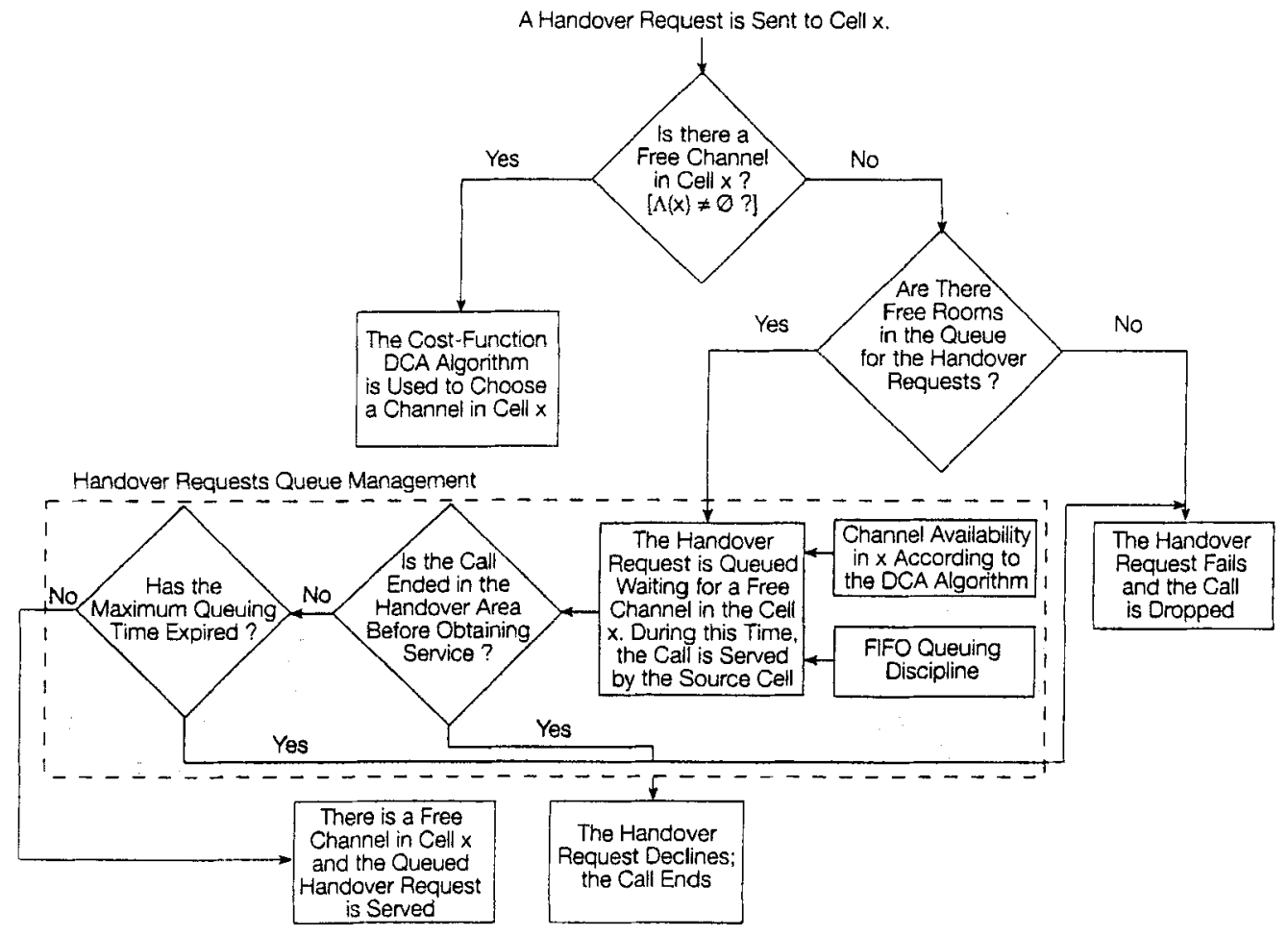

Fig. 5 - The policy used to manage the queued handover requests.

(5) The extension of the overlap area is a parameter that directly affects the handover queueing performance. Obviously, a wider overlap area leads to a lower handover failure probability, under specified traffic load conditions.
(') On the basis of the assumptions on mobility and overlap areas. the maximum queueing time $t_{\text {max }}$ is the same for all the handover requests, then, the most urgent request is also the oldest one (i.e.. FIFO policy). 
from the channel on which the call is actually ended, a channel rearrangement (i.e., an intra-beam handover) is performed in $x$. More details are given in reference [10].

\section{A CELLULAR NETWORK FOLDED ONTO ITSELF}

The simulated network is parallelogram shaped with a hexagonal layout; this is a common choice in the literature to study terrestrial cellular systems [22]. However, in order to adapt this topology to the specific characteristics of global-coverage MSSs, where the cellular network is three-dimensional, we define below a cellular network folded onto itself where each cell has a complete belt of interfering cells.

We denote by $N$ the number of cells per side in the cellular network. Each cell of the network has an identification number, $n=1,2,3, \ldots ., N^{2}$ (in Fig. $6, N=7$ ). Let us denote by $\Pi$ the set of cells that belong to the simulated parallelogram network: $\Pi=\left\{1,2,3, \ldots, N^{2}\right\}$. Moreover, an oblique reference with the origin in the center of the cell No. 1 is used, as shown in Fig. 6. If we normalize the distance among two adjacent cell centers, each cell center is denoted in this reference by a couple of integer numbers ( $\xi$, $\eta)$; both $\xi$ and $\eta$ belong to the set $\Delta=\{0,1,2, \ldots N-1\}$.

We assume that the belt of interfering cells of cell $x$ (i.e., $I(x)$ ) is formed by two tiers of adjacent cells [23]; then, the cells of the two more external orders in the parallelogram shaped network have an incomplete belt of interfering cells and form the set of "border" cells, $B$. Consequently, we name "central" cells those cells belonging to the set $\Pi-B$ (e.g., in Fig. 6, the cells No. $17,18,19,24,25,26,31,32,33)$.

Let us consider a generic cell $z \equiv\left(\xi_{-}, \eta_{-}\right)$on the border of the network; a generic cell $k \in I(z)$ can be identified as follows: $k \equiv\left(\xi_{z}+a, \eta_{z}+b\right)$, where $a, b \in\{-1$, $-2,0,1,2\}$ and $|a|+|b| \neq 0$ and $-2 \leq a+b \leq 2$.

- If $k \in \prod$ (i.e., both $\xi_{\tau}+a \in \Delta$ and $\eta_{z}+b \in \Delta$ ), the interfering cell $k$ really exists;

- Otherwise, if $k \notin \Pi$, the interfering cell $k$ becomes a dummy interfering cell of $z$ according to the following rule: the coordinates of the interfering cell $k=\left(\xi_{2}+a, \eta_{2}+b\right)$ are transformed into $k \equiv\left(\xi_{k}, \eta_{k}\right)$, where $\xi_{k}=T\left(\xi_{z}+a\right), \eta_{k}=T\left(\eta_{z}+b\right)$ according to the operator $T(v)$ defined below:

$T(v)= \begin{cases}N+v, & \text { if } v<0 \\ v, & \text { if } 0 \leq v<N \\ v-N, & \text { if } v \geq N\end{cases}$

Therefore, a cell on a border of the simulated network is virtually adjacent to and interferes with border cells on the other side of the network. In Fig. 6, the interfering cells of the border cell No. 48 are shown; note that the dashed cells are dummy.

When a folded network is used, an MS with a call in progress, which goes out from a side of the network.

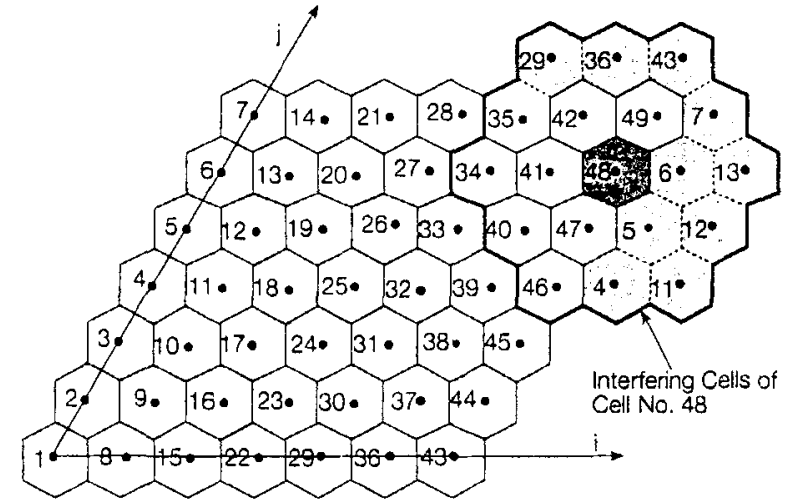

Fig. 6 - An example of dummy interfering cells for the parallelogram shaped cellular network, with 7 cells per side.

enters the network on the opposite side, thus ideally reproducing the behavior of an infinite network.

In our previous works $[9,10]$, the folded network was not adopted; then, central cells experienced more unfavourable interfering conditions with a DCA algorithm, because they had a complete belt of interfering cells. The blocking values were estimated only from central cells. Therefore, our previous comparisons among FCA and DCA techniques were based on the worst case. Now, the use of a folded network (where all the cells experience the same interfering conditions) permits to estimate the blocking probabilities from all the cells of the network, so achieving more reliable results. We have verified by simulation that the values of $P_{b 1}$ and $P_{b 2}$ are uniform all over the cellular network if a uniform traffic is considered: no border effect is experienced. This is a significant advantage when the performance of a channel allocation technique is evaluated.

If we use the cost function defined in (5) - (7) based on the regular FCA pattern and we consider an interfering belt formed by two tiers, the network can be correctly folded onto itself only for values of $N$ which are multiple of 7 (e.g., $N=7,14,28, \ldots$ ) owing to the parallelogram shape of the simulated network: only these special values of $N$ allow that the dummy interfering cells around a border cell have the same nominal FCA pattern used for the network. We have verified by simulation that the blocking results are insensitive to network sizes: the performance evaluation of the channel allocation techniques shown in this paper for $N=7$ (section 6) has been also carried out for $N=14$ and no difference has been found. This is another significant advantage of the folded network, because the blocking performance estimated on networks of reduced sizes is also valid for larger networks. This result allows to speed-up simulation runs.

\section{ESTIMATION OF THE SIGNALING LOAD}

The resource management techniques have been compared in the next Section in terms of the quality of service parameters (i.e., $P_{b 1}, P_{b 2}, P_{\text {drop, }}$ and $P_{n, s}$ ) that have been defined in section 1 and in terms of the signaling load to 
be supported by the network. The derivation of the blocking parameters has been extensively considered in the literature, and references $[10,17,19,20]$ can be used for more details. Then, this section focuses on the definition of a suitable measure for evaluating the average signaling load to be supported per call by the system; this is an important parameter from the network operator standpoint in order to study the practical feasibility and the implementation complexity of a resource management strategy.

Since the DCA techniques compared in this paper differ on the basis of both handover policies and channel rearrangement strategies to manage new call attempts, the signaling load has been expressed in terms of the average overhead, $\sigma$, to be supported per call on the air interface in order to carry out both intra-beam and inter-beam handover procedures. The events during call lifetime that contribute to $\sigma$ are taken into consideration by the specific counters that are summarized in Table 1, where we have used the following definitions:

$$
\begin{aligned}
& \phi \triangleq\left\{\begin{array}{l}
1, \text { if a channel rearrangement is performed } \\
0, \text { otherwise }
\end{array}\right. \\
& \psi \triangleq\left\{\begin{array}{l}
1, \text { if the handover procedure } \\
\text { is successfully performed } \\
0, \text { otherwise }
\end{array}\right.
\end{aligned}
$$

Note that in Table 1 we have distinguished the contributions $\phi$ for the different events that may require channel rearrangements: the contribution $\phi_{a}$, for the reconfiguration that may be needed to serve a new call attempt according to the PPA-DCA algorithm; the contribution $\phi_{h}$ for the rearrangement performed in the originating cell of a handover; the contribution $\phi_{r}$, due to the channel reconfiguration that may be required at the end of the call.

Parameter $\sigma$ is not an exact counter for the number of messages exchanged on the air interface between MS and satellites. Rather, it can be considered as a useful estimation (even if rough) of the overhead to be supported on the air interface due to the signaling procedures related to handovers and channel rearrangements. According to this, we consider that, for instance, a successful handover procedure with a channel rearrangement in the source cell causes a signaling load on the air interface three times greater than that due to a channel rearrangement at the end of a call.

The signaling load contributions in Table 1 have been

Table 1 - Contributions to $\sigma$ for each event during call lifetime

\begin{tabular}{|c|c|}
\hline Events & Contributions to $\sigma$ \\
\hline New call arrival & $\phi_{t}$ \\
\hline Handover request & $1+\phi_{t}+\psi$ \\
\hline Call end & $\phi_{r}$ \\
\hline
\end{tabular}

computed by assuming that channel rearrangements within a cell are procedures completely controlled and originated by the network and that inter-beam handovers are mobile controlled, make-before-break, backward procedures $\left({ }^{7}\right)$.

In the simulations, the average signaling load to be supported on the air interface per call, $\sigma$, has been obtained as the ratio between the signaling contributions computed according to Table 1 during a given time interval in all the cells of the system and the number of served calls in that interval.

Let us denote by $m$, the signaling load for a given call in order to manage channel rearrangements and inter-beam handover requests; then, $E[m]$ gives $\sigma$. Let us denote by $h_{o}$ the (random) number of inter-beam handover requests issued by an MS; note that if $h_{o}>0$, only the last handover request may be unsuccessful and cause the call dropping. In the following expression of $m$, parameter $\psi$ refers to the management of the last handover request:

$m=\phi_{a}+\sum_{i=1}^{h_{o}}\left(2+\phi_{h i}\right)-(1-\psi)+\psi \phi_{t} \frac{\text { overhead }}{\text { call admitted }}$

In order to obtain $\sigma$, we take the expectation on both sides of (11); then, we use the following average values, obtained according to $(10)$ :

$E\left[\phi_{a l}\right]=$ the probability that a channel rearrangement is performed in order to admit a new call attempt in the network (only with the PPADCA algorithm), $P_{r a}$

$E\left[\phi_{h i}\right]=$ the probability that a channel rearrangement is performed when a channel is released in the originating cell of the handover, $P_{r h}$

$E\left[\phi_{t}\right]=$ the probability that a channel rearrangement is performed when the call physically ends, $P_{r t}$

$E[\psi]=$ the probability that the last inter-beam handover is successfully performed; it is approximately equal to $1-P_{\text {drop, }}$, if we neglect the possibility that this handover request declines due to the reduced extension assumed for the overlap areas;

$E\left[h_{o}\right]=$ the average number of handover requests per call admitted into the network. $E\left[h_{0}\right]$ can be obtained from parameter $n_{h}$ (i.e., the average number of handover requests per call attempt) divided by $\left(1-P_{b 1}\right)$ in order to take account of the call attempts that are not accepted in the network.

For the channel rearrangement conditions, it is reasonable to admit that there is no difference between a

(7) A handover request message is issued by the active MS to the current sutellite when a handover criterion is met; if the handover is successful, the satellite sends back in message to the MS that is informed about the new channel to be used in the destination cell; rinally, a channel rearrangement (network controlled) may be required in the originating cell of the handover, according to the DCA de-allocation algorithin performed to release a channet in this cell. 
channel released in a cell due the physical end of the call or due to a handover towards an adjacent cell. Therefore, we consider that $P_{r h}=P_{r} ;$ then, in both cases we simply use the symbol $P_{r}$.

Finally, $\sigma$ results in:

$$
\begin{aligned}
\sigma= & P_{r a}+\frac{n_{h}}{1-P_{b 1}}\left(2+P_{r}\right)-P_{\text {drop }}+ \\
& \left(1-P_{\text {drop }}\right) P_{r} \frac{\text { overhead }}{\text { call admitted }}
\end{aligned}
$$

This formula permits to estimate the impact on the signaling load $\sigma$ of the degree of mobility $\alpha$ and the traffic load per cell $\lambda$. Note that $n_{h}=n_{h}\left(P_{b 1}, P_{b 2}, \alpha\right)$ and $P_{\text {drop }}=P_{\text {drop }}\left(P_{b 1}, P_{b 2}, \alpha\right)$ can be analytically expressed as shown in [10]. If the traffic load $\lambda$ increases (i.e., $P_{b 1}$ and $P_{b 2}$ increase), $P_{\text {drop }}$ increases and $n_{h}$ decreases; moreover, if the mobility increases (i.e., $\alpha$ decreases), $n_{h}$ and $P_{\text {drop }}$ increase.

Also the channel rearrangement probabilities $P_{r}$ and $P_{r a}$ depend both on $\alpha$ and $\lambda$. We expect that, for very low traffic loads per cell as well as for very high traffic

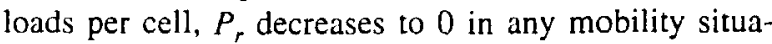
tion. The reason of this behavior is that, in these traffic conditions, each cell tends to use the channels according to the FCA pattern, then no channel reconfiguration is needed at the call termination in a cell. Whereas, we expect that $P_{r a}$ monotonically increases as the traffic load increases in any mobility condition: for low traffic loads per cell, $P_{r a}$ is close to 0 , because channel rearrangements are not required in order to admit new calls into the network; moreover, when the traffic congestion increases, channel rearrangements become more and more necessary in order to accept new calls.

Finally, as mobility increases (i.e., a decreases), we have a greater arrival rate of channel demands due to handover. In order to fulfil these demands, the regular channel allocation according to the FCA pattern is greatly impaired. Then, a greater number of channel rearrangements are required in order to compact the use of channels among the cells of the network. We may conclude that $P_{r}$ increases as mobility increases.

All these considerations will be validated by the simulation results shown in the next section.

According to the mobility assumptions, $n_{h} \simeq 1 / \alpha$ and $P_{\text {drop }}=0$ for low traffic loads per cell (i.e., $P_{b 1} \simeq P_{b 2} \simeq$ $0)$ [10]. Then, from (12), we can write the following expression of $s$ for low traffic loads:

$$
\begin{gathered}
\sigma\left(P_{b 1} \simeq 0, P_{b 2} \simeq 0\right) \simeq P_{r a}+ \\
\frac{1}{\alpha}\left(2+P_{r}\right)+P_{r} \frac{\text { overhead }}{\text { call admitted }}
\end{gathered}
$$

This value can be considered valid for any channel allocation technique in the traffic range where $P_{b 1} \simeq$ $P_{b 2} \simeq 0$ (note that $P_{r a}$ is equal to 0 , if the PPA algorithm is not used).

\section{Simulation RESUltS}

A simulation tool has been built according to the assumptions on user mobility (section 2 ) and overlap areas (sectrion 3 ) and on the basis of the topology chosen for the cellular network (section 4). The following values of the parameters have been used in the simulations considered in this section:

- the average arrival rate of new call attempts per cell, $\lambda$, varies from 2 to 3 calls $/ \mathrm{min} /$ cell (i.e., uniform traffic);

- the average call duration $T_{m}$ is equal to $3 \mathrm{~min}$;

- the product $T_{m} \cdot \lambda$ represents the "traffic intensity per cell due to new call arrivals": it varies from 6 to 9 erlang/cell;

- the belt of interfering cells is formed by two tiers of cells;

- the simulated cellular network is parallelogram shaped with 7 cells per side $(N=7)$;

- a number of 70 channels is available for the system; - both the IRIDIUM system ( $V_{t r k}=26,600 \mathrm{~km} / \mathrm{h}$, $R=212.5 \mathrm{~km}$; then, if $\left.T_{m}=3 \mathrm{~min}, \alpha \simeq 0.32\right)$ and a GEO-MSS ( $\alpha \rightarrow \infty$, users are "fixed") are considered;

- an infinite capacity is assumed to queue the handover requests that do not obtain immediately service;

- the maximum queueing time is $t_{w \max }=\alpha T_{m} / 10=$ $0.1 \mathrm{~min}$ for the IRIDIUM system.

Since from the user standpoint, the dropping of a call in progress is less desirable than the blocking of a new call attempt, a high quality of service is obtained if handover requests are prioritized with respect to new call attempts. The handover prioritization is obtained at the expenses of an increased value for the blocking probability of new call attempts; this is a drawback from the operator standpoint. Therefore, an efficient channel allocation technique has to be selected that is able to attain a low value of the handover failure probability (i.e., $P_{b_{2}}$ ), without excessively increasing the blocking of new call attempts (i.e., $P_{b l}$ ). We have assumed that a good tradeoff between these "opposite" needs is obtained by the following target values that are valid for high mobility systems such as LEO-MSSs: $P_{b 1} \leq 10^{-2}$ and $P_{b 2} \leq 5$. $10^{-4}$; that is, we assume that there is (at least) an order of magnitude of difference between the requirement on $P_{b 1}$ and the requirement on $P_{b 2}$; these values can be considered as realistic figures for next generation MSSs.

In the following, we will denote by DCA the basic dynamic channel allocation technique with no queueing for handover requests, by DCA-QH the same allocation technique with the queueing of handover requests and by PPA-DCA-QH the algorithm proposed in this paper with a PPA policy to manage new call attempts and the queueing of handover requests.

The comparison among DCA, DCA-QH and PPADCA-QH techniques in the IRIDIUM mobility case 


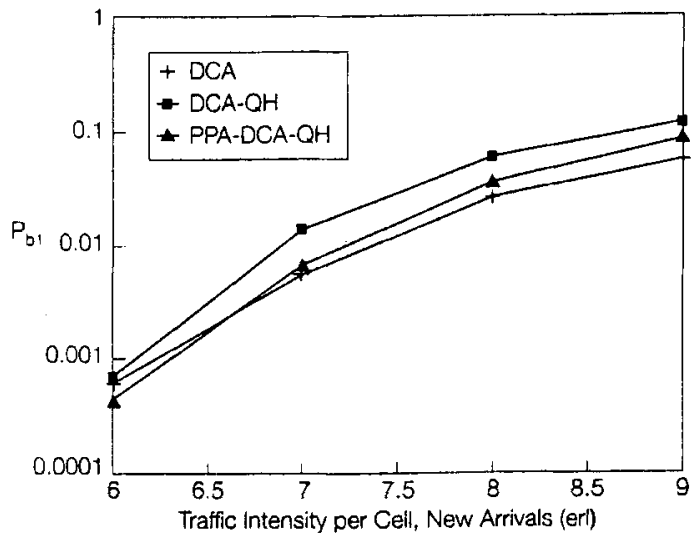

Fig $7-P_{b 1}$ performance of DCA, DCA-QH and PPA-DCA-QH in the IRIDIUM mobility case.

$(\alpha \simeq 0.32)$ is shown in Figs. 7, 8 and 9 for $P_{b 1}, P_{b 2}$ and $P_{n s}$, respectively. We can note that the performance of the DCA technique is not satisfying due to the high values of $P_{b 2}$ all over the traffic range under consideration. A possible solution to decrease these values of $P_{b 2}$ is achieved by the queueing of handover requests. Then, we consider the DCA-QH technique that privileges the handover service: $P_{b 2}$ is reduced at the expenses of an increased value of $P_{b 1}$. Finally, it is possible to mitigate this increase in $P_{b 1}$ by resorting to the PPA-DCA-QH technique: this solution improves the quantity of traffic admitted into the network by accepting a little increase in $P_{b 2}$. Therefore, the PPA-DCA-QH strategy can represent a good compromise between users' needs and operator's needs.

The requirements on $P_{b 1}$ and $P_{b 2}$ are fulfilled by $\mathrm{DCA}-\mathrm{QH}$ for a traffic intensity of new call attempts per cell of about 6.8 erlang/cell, whereas by PPA-DCA-QH for 7 erlang/cell.

The $P_{n s}$ performance for DCA, DCA-QH and PPADCA-QH techniques is presented in Fig. 9. Moreover, for the sake of completeness, the results for FCA and FCA$\mathrm{QH}$ are also shown in this graph. We can note that the PPA-DCA-QH strategy achieves the best performance.

The behavior of the number of inter-beam handover requests per call, $n_{h}$, as a function of the traffic intensity

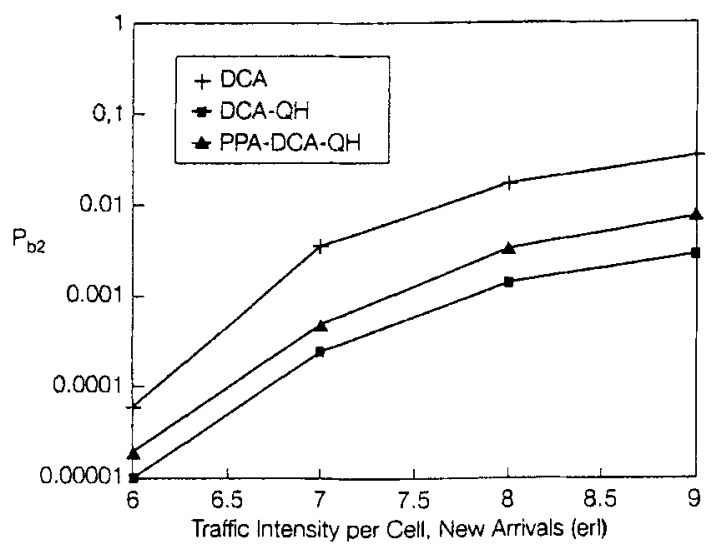

Fig. 8 - $P_{b 2}$ performance of DCA, DCA-QH and PPA-DCA-QH in the IRIDIUM mobility case.

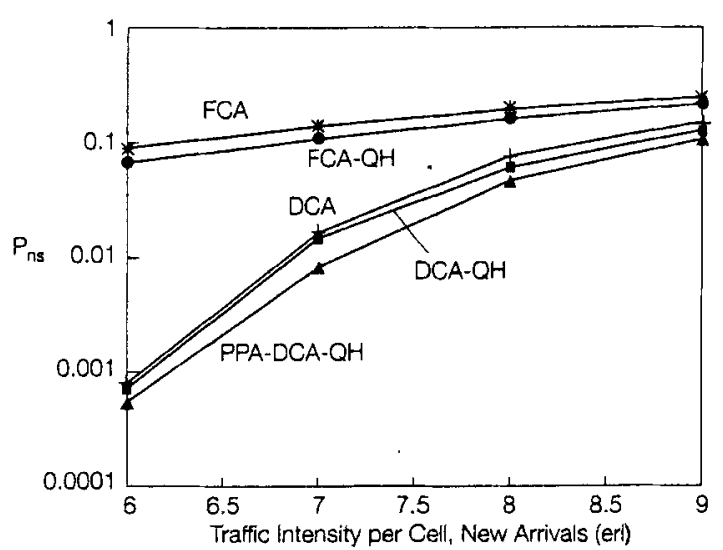

Fig. 9 - $P_{n s}$ performance of DCA, DCA-QH and PPA-DCA-QH in the IRIDIUM mobility case.

per cell due to new call attempts is shown in Fig. 10 for DCA, DCA-QH and PPA-DCA-QH in the IRIDIUM case. We can note that, in all cases, $n_{h}$ decreases as the traffic load increases. This behavior is due to the fact that the increase of traffic causes an increase in the blocking probability for handover requests (Fig. 8); then, less handover requests are performed on average per call. Moreover, for low traffic loads $n_{h}$ approaches $1 / \alpha \simeq 3.12$ handovers/call for all the techniques.

Fig. 11 shows the Pns performance for DCA, PPADCA and FCA techniques in the GEO-MSS case, where the users have been assumed "fixed" $(\alpha \rightarrow \infty)$. Now, $P_{n s}=P_{b l}$, because there is no handover procedure during call lifetime. The blocking results are much lower than those shown in Fig. 9 for the IRIDIUM mobility case at the same traffic load per cell. This difference highlights how much mobility affects the performance of a channel allocation technique. The requirement on $P_{b 1}$ is fulfilled by DCA for 7 erlang/cell and by PPA-DCA for 7.25 erlang/cell. Finally, in Fig. 11 the performance of other DCA techniques found in the literature (namely, LODA and $\mathrm{BCO}$ [22]) and a lower bound for the performance of DCA techniques [14] have been also shown.

The comparisons among the techniques in terms of $\sigma$ are shown in Figs. 12 and 13 for the GEO case and for

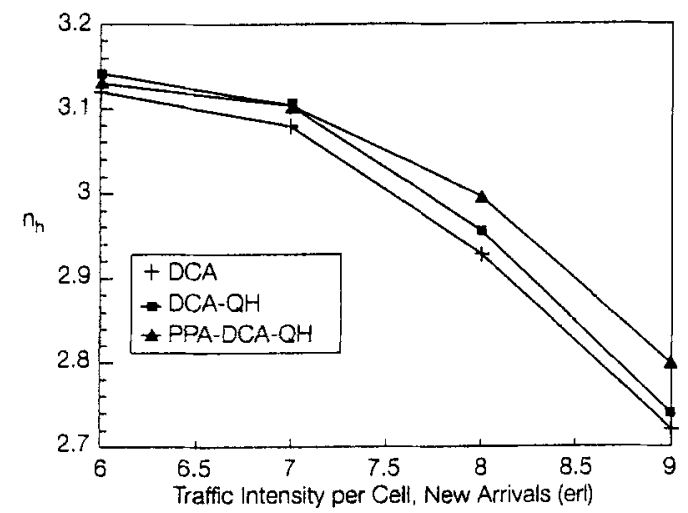

Fig. 10 - Behavior of $n_{i}$ as a function of the traffic intensity due to new call arrivals per cell, for DCA. DCA-QH and PPA-DCA-QH in the IRIDIUM mobility case. 


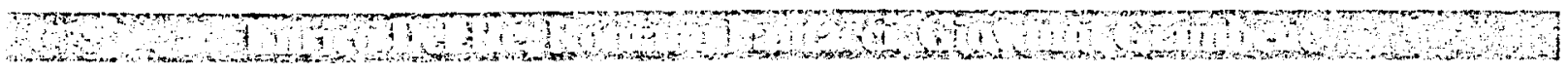

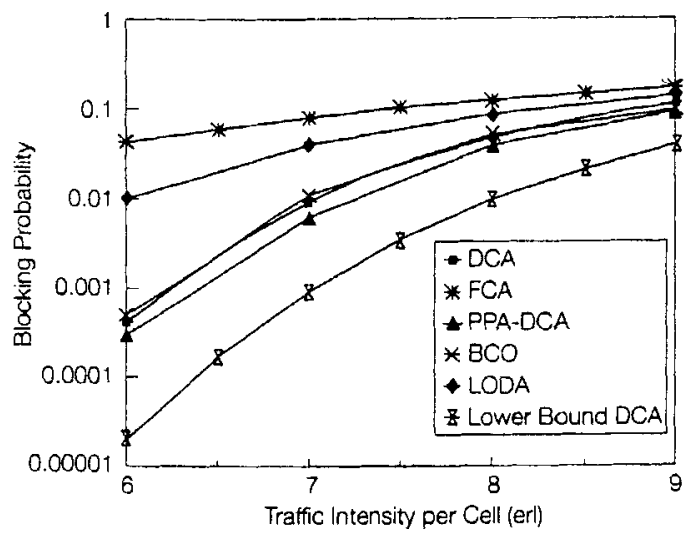

Fig. 11 - $P_{\text {a }}$ performance of BCO, LODA, FCA, DCA and PPADCA for GEO-MSSs with a lower bound for the DCA performance.

the IRIDIUM case, respectively. The question to be investigated here is whether the introduction of the PPA-DCA strategy causes a significant increase in the signaling load with respect to the DCA algorithm due to the channel rearrangements that may be required to serve new call arrivals. Let us comment the results shown in Fig. 12, where a GEO-MSS is considered.

- Since the users have been supposed "fixed", the only contribution to $\sigma$ in the DCA case is due to the channel rearrangements performed at the call termination instants. In this case, $\sigma$ is given by the probability that a termination requires a channel rearrangement (i.e., $P_{r}$ ). The behavior of $\sigma$ denotes a slight dependence on the traffic load; $\sigma$ decreases both for low and high traffic loads per cell.

- In the PPA-DCA case, $\sigma$ increases when the traffic load increases. The different behavior of $\sigma$ with respect to DCA depends on the channel rearrangements performed at the call arrival instants: if the cell congestion increases, it becomes more likely that a channel reconfiguration is needed in order to admit a new call in the network. The difference between PPA-DCA and DCA is due to the probability that a channel rearrangement is required to serve a new call arrival (i.e., $P_{r a}$ ). Note that the signaling load difference is close to 0 for

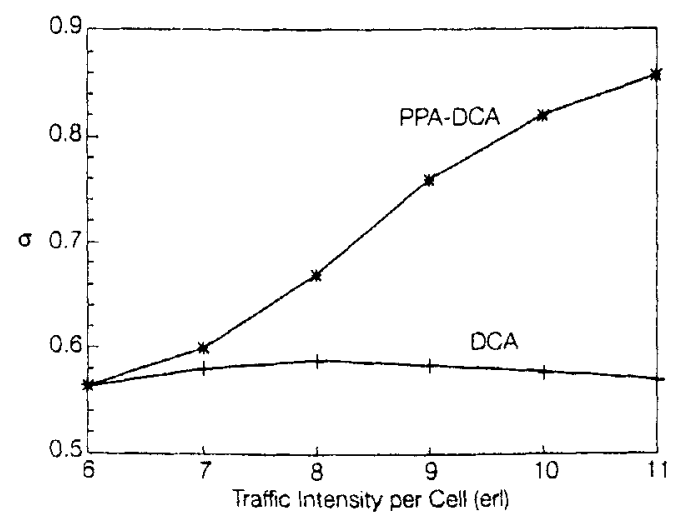

Fig. 12 - Behavior of $\sigma$, in the GEO cuse for DCA and PPA-DCA.

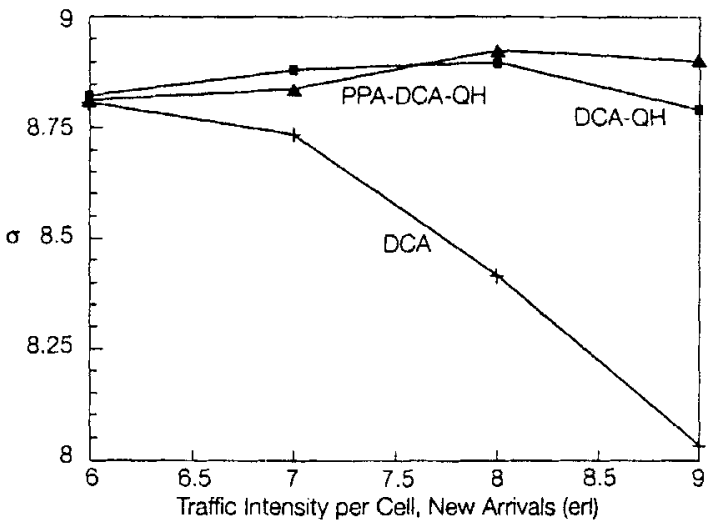

Fig. 13 - Behavior of $\sigma$, in the IRIDIUM case for DCA, DCA-QH and PPA-DCA-QH.

low traffic loads per cell; moreover, this difference is extremely reduced (i.e., less than 0.10 ) in the traffic region of interest $\left(^{8}\right)$.

- For low traffic loads per cell (i.e., $\lambda T_{m} \leq 6$ erlang/cell), both PPA-DCA and DCA give $\sigma=$ 0.58 overhead/call. According to (13) with $\alpha \rightarrow \infty$, we may consider that $P_{r u} \approx 0$ and $\sigma \approx P_{r}$.

Let us refer to Fig. 13, where DCA, DCA-QH and PPA$\mathrm{DCA}-\mathrm{QH}$ are compared in the IRIDIUM mobility case. Note that the values of $\sigma$ are much greater in the IRIDIUM case $(\sigma \simeq[8,9]$ overhead/call) than in the GEO case $(\sigma<1$ overhead/call); this is exclusively due to mobility differences. The following comments are in order:

- As for the DCA technique, $\sigma$ decreases when the traffic load increases, because the handover failure probability, $P_{b 2}$, increases and, then, the average number of handover procedures per call decreases (Fig. 10). Moreover, we have verified by simulation that $P_{r}$ values are close to 0.62 all over the examined traffic range.

- In the DCA-QH case, the reduced values of $P_{b 2}$ cause an increased number of handovers per call (Fig. 10) and, then, higher values for $\sigma$. Moreover, simulations have shown that $P_{r}$ values slightly increase from 0.61 to 0.63 from 6 to 9 erlang/cell.

- Let us refer to the PPA-DCA-QH case. If the traffic load per cell increases, we have two opposite trends: on one hand, the contribution to $\sigma$ due to inter-beam handovers reduces, because less handovers are managed per call due to the increase in $P_{b 2}$ (Fig. 10); on the other hand, the number of channel rearrangements needed to admit new calls in the network increases (i.e., it is more likely that a new call is accepted in the network by a channel rearrangement). The result is a behavior slightly different from that of DCA-QH.

(") The "traffic region of imterest" for a given channel allocation tech nique is that region where $P_{b 1} \leq 10^{-2}$ and $P_{b, 2} \leq 5 \cdot 10^{-1}$. 


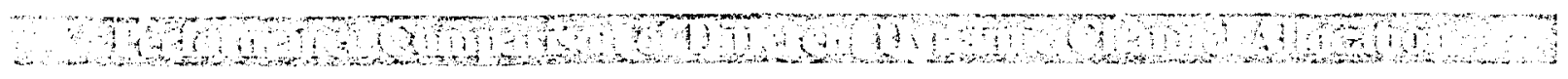

- For low traffic loads per cell (i.e., $\lambda T_{m} \leq 6$ erlang/cell), both PPA-DCA-QH, DCA-QH and DCA give $\sigma \simeq 8.8$ overhead/call. Then, according to (13), $P_{r a}=0$ and $P_{r}$ values are almost the same for all the techniques: $P_{r}=0.62$.

According to the traffic operating point selected for DCA-QH and PPA-DCA-QH (6.8 erlang/cell and 7 erlang/cell, respectively), we obtain from the graph in Fig. 13 that the signaling load with DCA-QH is greater than that of PPA-DCA-QH (i.e., 8.88 overhead/call and $8.82 \mathrm{overhead} /$ call, respectively). This interesting result confirms that PPA-DCA-QH is an attracting solution, because it improves network performance (in terms of $\left.P_{n s}\right)$ without increasing the signaling load.

\section{CONClusions}

In this paper, resource management techniques suitable for applications in MSSs have been proposed in order to cope with the increasing need of mobile satellite communications. Two different mobility scenarios have been envisaged: both GEO- and LEO-MSSs.

A simulation tool has been built in order to allow the comparison among several resource management strategies with different conditions for mobility and traffic loads. The performance of channel allocation techniques has been expressed in terms of blocking probabilities (for new call attempts and handover requests) and signaling load.

Different dynamic channel allocation techniques based on the evaluation of a cost function, have been compared. Moreover, in the LEO-MSSs case, the queueing of handover requests has been considered essential in order to reduce the handover failure probability. A particularly interesting DCA solution has been selected, called PPADCA-QH, where a new call arrival is accepted by the network, even if there is no available channel, by allowing a channel rearrangement in an interfering cell. This technique has shown a good performance both in terms of blocking probabilities and signaling load.

\section{Acknowledgements}

The authors wish to thank the referees for providing helpful suggestions.

\section{Manuscript received on September 18, 1996.}

\section{REFERENCES}

(1) D. C. Cox: Wireless personal communications: what is it? "IEEE Personal Communications", April 1995. p. 20 - 35.

[2] Z. Haas: Mobile communication netwerks. [EEE GLOBECOM ${ }^{\circ} 94$ Tutorial n. 3, S.Francisco, California. November 28, 1994.
[3] J. F. Huber: System alternatives - satellites and network aspects First European Workshop on Mobile/Personal Satcoms, Pane Discussion B, Frascati, Italy, October 14, 1994.

[4] E. Del Re: A coordinated european effort for the definition of a satellite integrated environment for future mobile communica tions. "IEEE Communications Magazine", Vol. 34, No. 2. February 1996, p. $98-104$

[5] E. Del Re Edit.: Mobile and personal communications. Proceeding of the 2-nd Joint COST 227/231 Workshop. Elsevier Science 1995

[6] F. Ananasso, F. Delli Priscoli: The role of satellites in persona communication services. "IEEE Journal on Selected Areas in Comm.", Vol. 13, No. 2, February 1995, p. $180-196$.

[7] A. Ganz, Y. Gong, Bo Li: Performance Study of low earth-orbit satellite systems. "IEEE Trans. on Comm.", Vol. 42, No. 2/3/4, February/March/April 1994, p. 1866 - 1871

[8] G. Maral, J-J. De Ridder, B.G. Evans, M. Richharia: Low earth orbit satellite systems for communications. "Int. J. Sat. Comm.", Vol. 9, 1991, p. 209-225.

[9] E. Del Re, R. Fantacci, G. Giambene: Performance analysis of a dynamic channel allocation technique for satellite mobile cellular networks. "International Journal of Satellite Communications", Vol. 12, January/February 1994, p. 25-32.

[10] E. Del Re, R. Fantacci, G. Giambene: Efficient dynamic channel allocation techniques with handover queuing for mobile satellite networks. "IEEE Journal on Selected Areas in Commun.", Vol. 13, No. 2, February 1995, p. $397-405$.

[11] E. Del Re, R. Fantacci, G. Giambene: An efficient technique for dynamically allocating channels in satellite cellular networks. Proc of IEEE GLOBECOM'95, Singapore, November 13-17, 1995 , p. $1624-1628$

[12] J. E. Padgett, C. G. Gunter. T. Hattori: Overview of wireless personal communications. "IEEE Communications Magazine", Vol. 33, No. 1, January 1995, p. 28 - 41.

[13] Gregory P. Pollini: Trends in handover design. "IEEE Communications Magazine", Vol. 34, No. 3, March 1996 p. $82-90$.

[14] L. J. Cimini, G. J. Foschini, Chih-Lin I, Z. Miljanic: Call block ing performance for dynamic channel allocation in microcells. "IEEE Trans. on Comm.", Vol. 42, No. 8, August 1994 p. $2600-2607$

[15] G. P. Pollini, K. S. Meier-Hellstern, D. J. Goodman: Signalling traffic volume generated by mobile and personal communications. "IEEE Communications Magazine", Vol. 33, No. 6, June 1995. p. $60-65$.

[16] J. Foschini, B. Gopinath, Z. Miljanic: Channel cost of mobility. "IEEE Trans. on Veh. Tech.", Vol. 42, No. 4, November 1993 , p. $414-424$

[17] D. Hong. S. S. Rappaport: Traffic model and performance anatysis for cellular mobile radio telephone systems with prioritized and nonprioritized handoff procedures. "IEEE Trans. on Veh. Tech.", Vol. VT-35, No. 3, August 1986, p. 77 - 92.

[18] S. Tekinay, B. Jabbari: Handover and channel assignments in mobile cellular networks. "IEEE Communications Magazine", Vol. 29, No. 11, November 199 !, p. $42-46$.

[19] S. Tekinay, B. Jabbari: A measurement-based prioritization scheme for handovers in mobile cellular networks. "IEEE J. Selected Areas Comm.", Vol. 10, No. 8, October 1992, p. 1343 1350.

[20] Yi-Bing Lin, S. Mohan. A. Noerpel: Queuing priority channel assignment strategies for pcs hand-off and initial access. "IEEE Trans. on Veh. Tech.", Vol. 43, No. 3, August 1994, p. 704-712.

[21] E. Del Re, R. Fantacci, G. Giambene: Radio resource management. SAINT Project, Deliverable D11. R2117-FIUDIE.DR.P211-B1, September 1995

[22] M. Zhang, P. Tak-Shing Yum: Comparisoms of channel-assignment strategies in cellular mobile telephone systems. "IEEE Trans. on Veh. Tech.", Vol. 38, No. 4, November 1989, p. 211. 215.

[23] V. H. MacDonald: The cellular concept. "Bell Syst. Tech. J.", Vol. 58, January 1979 , p. $15-41$. 